\title{
Straw Lives (After the Fires)
}

\section{Susan Adams}

burnt blood

blackens sky

burdened with ash

we inhale

our history

to stay alive

tears scar

soot skin

tattoo pain

leave hollow

orphans with

handicapped lives

futures die

beat empty dreams

beat and break

parchment is tinder

memory the epitaph

that reminds us 